\title{
Impacts of the Spaceflight to the Immune System
}

\author{
Doston Sultonov, M.D. ${ }^{1,2}$, Young Hyo Kim, M.D., Ph.D. ${ }^{1,2}$ \\ ${ }^{1}$ Inha Institute of Aerospace Medicine, ${ }^{2}$ Department of Otorhinolaryngology, Head and Neck Surgery, Inha University \\ School of Medicine, Incheon, Korea
}

Received: October 25, 2021

Revised: November 22, 2021

Accepted: November 22, 2021

Corresponding Author

Young Hyo Kim

Department of Otorhinolaryngology, Head and Neck Surgery, Inha University School of Medicine, 27 Inhang-ro, Jung-gu, Incheon 22332, Korea

Tel: $+82-32-890-2437$

Fax: +82-32-890-3580

E-mail: inhaorl@inha.ac.kr

ORCID

https://orcid.org/0000-0002-3623-1770

*This study was supported by the Ministry of Education, Science, and Technology through the National Research Foundation of Korea (no. NRF2018R1A6A1A03025523).

\begin{abstract}
Changes the gravity has a significant affect on the immune system. Astronauts experience the gravity changing during spaceflight, especially when launching and landing they experience hypergravity, and during spaceflight they feel microgravity. Both hypergravity and microgravity has an impact to the immune system, but not the same effect. These impacts have been investigated extensively during spaceflight in astronauts and in model experiments conducted on Earth as well. Astronauts during spaceflight feel the hypergravity, psychological stress, fear, high doses of radiation and microgravity. All these factors and changes may affect immune system directly or indirectly.
\end{abstract}

Keywords: Immune system, Aerospace medicine, Hypergravity, Altered gravity, Lymphopoiesis

\section{INTRODUCTION}

It is extremely difficult to perform experiments in real space. Therefore, several ground-based models have been developed to stimulate space environment. One of the most widely accepted models for stimulating hypergravity is the centrifugal device. By using centrifugal force due to the rotation, we could expose cultured cells or experimental animals to higher gravity than $1 \mathrm{G}$ [1]. Gravity is ubiquitous and influences tissue mechanical environment by affecting cell weight, extracellular hydrostatic pressure and fluid convection. By stimulating the presence of intense gravitational vectors, for instance with the aid of large diameter centrifuges, useful insight on cellular physiology can be gained and even exploited to elaborate novel therapies [2].

\section{EFFECTS OF HYPERGRAVITY}

Firstly, we want to say about hypergravity affects to the immune system in the following sections. Hypergravity impacts on the immune system are controversial and needs its solution. Research on the impact of hypergravity on the immune system is still limited. Some studies have evaluated changes in the mitogen-induced proliferation of lymphocytes, titers of several cytokines and subpopulations of the lymphocytes [1]. Concerning cell migration, hypergravity determines earlier human $\mathrm{T}$ lymphocyte motility on fibronectin-coated surfaces compared to normal gravity [3]. This in turn, gives possible impact on immunity of space crews. Moreover, hypergravity enhances human dendritic cell ability of activating lymphocyte $\mathrm{T}$ proliferation an adhesion (over 85\%) to human vascular smooth muscle cells.

Concerning its potential use as a therapeutic approach 
in pathological conditions due to infection, hypergravity ( $4 \mathrm{G}$ ) is reported to enhance survival of drosophila flies infected with the pathogenic fungus (Beauveria bassiana). Experiments also demonstrate that hypergravity improves resistance to Toll-mediated fungal infections [4]. The studies of hyprtgravity impacts on different animal models under pathological conditions induced by bacterial infection are strongly needed prior to envision a realistic application of hypergravity as a therapeutic approach [2].

Applied hypergravity induced a decrease in transepithelial electrical resistance without significant loss of cellular metabolic activity, weakening of the cell barrier function and increasing the cellular permeability of polar molecules in nasal mucosa. These effects of hypergravity could be used as a novel strategy for enhancing nasal drug delivery efficiency through temporal weakening of the cell barrier function [5].

Jang et al. [6] studied hormetic effect of hypergravity in a mouse model of allergic asthma and rhinitis, and according to their review of literature, there are no studies has been conducted on the effect of hypergravity on animals with allergic disorders. And they observed significant improvement of allergic airway inflammation in their clinical course. To establish the physiologic mechanisms underlying this clinical improvement, authors evaluated serum total and ovalbumin (OVA)-specific immunoglobulin $\mathrm{E}$ (IgE), expression of genes for interleukin (IL)-1 $\beta$, IL4, IL-5, IL-6, IL-10 and interferon- $\gamma$, inflammatory cells in bronchoalveolar nlavage (BAL) in mouse's lung homogenate. Results showed that, rotatory stimulus and chronic hypergravity caused a significant decrease of serum total IgE, inflammatory cells in BAL fluid. Regarding OVAspecific IgE, there was no significant decrease.

Chronic hypergravity also caused upregulation of for IL-1 $\beta$ and downregulation of IL- 4 and IL-10 in lung homogenate. Although the expression of IL- 5 showed some tendency to decrease in asthma/hypergravity group. The most interesting aspect of this study was that this study is the first to demonstrate the hormetic immune effect in animals with allergic disorder. However, the serum total IgE level showed significant decrease in asthma/ hypergravity group, as well as in asthma/rotatory stimuli group total IgE also significantly decreased. It might be the result of a stress response to rotatory stimuli. Another interesting aspect of this research is that, Jang et al. [7] suggested that changes in the allergic response in animals with allergic disorders may be unrelated to IgE-related mechanisms, because of the study showed no significant change in serum OVA-specific IgE after exposure short term hypergravity (10 G for 4 hours).

Histopathologic examination of lung parenchyma and nasal mucosa to confirm the affect of chronic hypergravity on the allergic inflammatory response. And they confirm histologically that chronic hypergravity could alleviate allergic asthma and rhinitis [7].

Furthermore, one of the hypotheses about the mechanism of hormetic effect of chronic hypergravity is that protection and repair mechanisms against stress could be involved. Because of increased metabolic rate due to increased weight, there could be more oxidative damage as a result. Therefore, activities of several antioxidant enzymes could be upregulated to protect an organism against this oxidative damage [2]. The study of the expression of several genes related with anti-oxidative and anti-apoptotic function also confirmed that after exposure chronic hypergravity significantly suppressed antiapoptotic factor and significantly enhanced proapoptotic enzymes, which are responsible for increasing apoptosis of inflammatory cells in the lung and nasal cavity. A decrease of inflammatory cells, in turn, could be responsible for the decreased Th2 cytokines, caused reduced allergic symptoms.

In short, according to the recent studies, we may say that chronic hypergravity might be increase an immune function in animals with allergic disorders. The biological responses of tissues in organism to hypergravity is motivated by the possibility to expose whole bodies to hypergravity conditions in order to achieve therapeutic goals [2].

\section{EFFECTS OF MICROGRAVITY}

During spaceflight astronauts feel the hypergravity more when the spacecraft launching and landing. However, during the space they experience the microgravity, high doses of radiation and psychological stress as well. The space radiation and stress may directly and in a stress hormone-dependent manner affect to the immune response of organism, especially acquired immune system [8].

In the acquired immune system plays the main role is lymphocytes. Antigen-specific T cells and B cells are con- 
verted into immunological memory cells that contribute effective immunity against pathogens [9]. The factors during spaceflight, which we mentioned above may affect the initial development of lymphocytes, as well as the development of lymphocytic immunological memory and herewith destroy acquired immune system.

Lymphopoiesis, differentiation and maturation of lymphocytes, myeloid cells, hematopoietic stem cells and mesenchymal stem cells (MSCs) occur in bone marrow. In turn, microgravity impacts on the bone marrow during spaceflight, and influenced to the differentiation and maturation of the stem cells. MSCs are the sources of osteoblasts, chondrocytes and adipocytes. Long-term being in the low gravitational condition without gravitational stimulation of their bones exhibit reduce in bone mass and immune function [8]. However, the role of microgravity in the MSCs dysfunction has not yet fully understood. Although, you may see the information or diagrams of mechanism about the alteration in differentiation of MSCs by microgravity and space radiation. Previous research suggested that in mice the space radiation which is a major environmental stressor in humans in space, may affect MSCs differentiation into adipocytes [10]. Although, Bcell frequency was reportedly not changed immediately after space flight, it was significantly reduced 1 week after landing [11]. This means that, spaceflight did not affect the immunoglobulin repertoires of mice after short-time spaceflight $[8,11]$.

Furthermore, besides the B-cells, also the T-cells output which are generated in thymus is reduced after spaceflight, suggesting impairment of T-cell development in the thymus $[8,12]$.

In addition, to human sample analysis, the influence of spaceflight on the thymus has been investigated in mice. The study performed in early stages that during 2 weeks of study the spaceflight caused an involution of the murine thymus [13]. In long-term spaceflight study also showed that also reduction of murine thymus $[8,14]$. This is suggested that spaceflight induces a thymic atrophy, however it is difficult to decide what factors affected to the thymic atrophy during spaceflight.

In turn, the spaceflight effects on immune system development and reducing it, leads to the changes in immune responses of the organism. In a study in the early 1980s reported that after spaceflight were reduced eosinophils and elevated neutrophils in the peripheral blood of astro- nauts [15]. Further studies revealed that spaceflight affects various immune parameters, such as the distribution of leukocytes, granulocyte and monocyte function, natural killer cell function, cytokine levels in plasma and in response to stimuli [16-18].

\section{CONCLUSION}

The gravity changes and environmental changes are one of the typical conditions during spaceflight, each factor directly or indirectly via induction of stress hormones may disturb immune system. Experimental methods incorporating low-gravity environments, for instance parabolic flight, simulated microgravity condition such as clinostat, long-term bed rest and hind limb suspension in experimental animals have been employed. It is necessary to carefully consider the extent to which these experimental methods reflect microgravity.

\section{CONFLICTS OF INTEREST}

No potential conflict of interest relevant to this article was reported.

\section{ORCID}

Doston Sultonov, https://orcid.org/0000-0001-6102-4450

Young Hyo Kim, https://orcid.org/0000-0002-3623-1770

\section{AUTHOR CONTRIBUTIONS}

Conceptualization: YHK. Data curation: DS. Analysis and interpretation: DS. Writing the original draft: all authors. Critical revision of the article: YHK. Final approval of the article: all authors. Overall responsibility: YHK.

\section{REFERENCES}

1. Jang TY, Jung AY, Kim YH. Hormetic effect of chronic hypergravity in a mouse model of allergic asthma and rhinitis. Sci Rep 2016;6:27260. https://doi.org/10.1038/srep27260

2. Genchi GG, Rocca A, Marino A, Grillone A, Mattoli V, Ciofani G. Hypergravity as a tool for cell stimulation: implications in biomedicine. Front Astron Space Sci 2016:3:26. https://doi.org/10.3389/fspas.2016.00026

3. Galimberti M, Tolić-Nørrelykke IM, Favillini R, Mercatelli R, Annunziato F, Cosmi L, et al. Hypergravity speeds up 
the development of T-lymphocyte motility. Eur Biophys J 2006;35:393-400. https://doi.org/10.1007/s00249-0060046-x

4. Taylor K, Kleinhesselink K, George MD, Morgan R, Smallwood T, Hammonds AS, et al. Toll mediated infection response is altered by gravity and spaceflight in Drosophila. PLoS One 2014;9:e86485. https://doi.org/10.1371/journal. pone.0086485

5. Kim D, Kim YH, Kwon S. Enhanced nasal drug delivery efficiency by increasing mechanical loading using hypergravity. Sci Rep 2018;8:168. https://doi.org/10.1038/s41598017-18561-x

6. Jang TY, Jung AY, Kwon S, Kim YH. Hypergravity enhances the therapeutic effect of dexamethasone in allergic asthma and rhinitis animal model. PLoS One 2018;13:e0197594. https://doi.org/10.1371/journal.pone.0197594

7. Jang TY, Kim KS, Park CS, Lim J, Huh KC, Heo MJ, et al. Exposure to hypergravity increases serum interleukin -5 and pulmonary infiltration in mice with allergic asthma. Cent Eur J Immunol 2014;39:434-440. https://doi.org/10.5114/ ceji.2014.47725

8. Mizoguchi T, Pinho S, Ahmed J, Kunisaki Y, Hanoun M, Mendelson A, et al. Osterix marks distinct waves of primitive and definitive stromal progenitors during bone marrow development. Dev Cell 2014;29:340-349. https://doi. org/10.1016/j.devcel.2014.03.013

9. Akiyama T, Horie K, Hinoi E, Hiraiwa M, Kato A, Maekawa $\mathrm{Y}$, et al. How does spaceflight affect the acquired immune system? NPJ Microgravity 2020;6:14. https://doi. org/10.1038/s41526-020-0104-1

10. Tascher G, Gerbaix M, Maes P, Chazarin B, Ghislin S, Antropova E, et al. Analysis of femurs from mice embarked on board BION-M1 biosatellite reveals a decrease in immune cell development, including B cells, after 1 wk of recovery on Earth. FASEB J 2019;33:3772-3783. https://doi. org/10.1096/fj.201801463R
11. Benjamin CL, Stowe RP, St John L, Sams CF, Mehta SK, Crucian BE, et al. Decreases in thymopoiesis of astronauts returning from space flight. JCI Insight 2016;1:e88787. https://doi.org/10.1172/jci.insight.88787

12. Gridley DS, Mao XW, Stodieck LS, Ferguson VL, Bateman TA, Moldovan M, et al. Changes in mouse thymus and spleen after return from the STS-135 mission in space. PLoS One 2013;8:e75097. https://doi.org/10.1371/journal. pone.0075097

13. Novoselova EG, Lunin SM, Khrenov MO, Parfenyuk SB, Novoselova TV, Shenkman BS, et al. Changes in immune cell signalling, apoptosis and stress response functions in mice returned from the BION-M1 mission in space. Immunobiology 2015;220:500-509. https://doi.org/10.1016/ j.imbio.2014.10.021

14. Taylor GR, Dardano JR. Human cellular immune responsiveness following space flight. Aviat Space Environ Med 1983;54(12 Pt 2):S55-S59.

15. Crucian B, Stowe RP, Mehta S, Quiriarte H, Pierson D, Sams C. Alterations in adaptive immunity persist during long-duration spaceflight. NPJ Microgravity 2015;1:15013. https://doi.org/10.1038/npjmgrav.2015.13

16. Stowe RP, Sams CF, Mehta SK, Kaur I, Jones ML, Feeback DL, et al. Leukocyte subsets and neutrophil function after short-term spaceflight. J Leukoc Biol 1999;65:179-186. https://doi.org/10.1002/jlb.65.2.179

17. Crucian BE, Zwart SR, Mehta S, Uchakin P, Quiriarte HD, Pierson D, et al. Plasma cytokine concentrations indicate that in vivo hormonal regulation of immunity is altered during long-duration spaceflight. J Interferon Cytokine Res 2014;34:778-786. https://doi.org/10.1089/jir.2013.0129

18. Mehta SK, Crucian BE, Stowe RP, Simpson RJ, Ott CM, Sams CF, et al. Reactivation of latent viruses is associated with increased plasma cytokines in astronauts. Cytokine 2013;61:205-209. https://doi.org/10.1016/j.cyto.2012.09.019 